\title{
METTL14 benefits the mesenchymal stem cells in patients with steroid-associated osteonecrosis of the femoral head by regulating the m6A level of PTPN6
}

\author{
Cheng Cheng ${ }^{1,}{ }^{*}$, Haoping Zhang ${ }^{2,}$, Jia Zheng ${ }^{1}$, Yi Jin' ${ }^{1}$, Donghui Wang ${ }^{1}$, Zhipeng Dai ${ }^{1}$ \\ ${ }^{1}$ Department of Orthopedics, Henan Provincial People's Hospital, Zhengzhou, Henan, China \\ ${ }^{2}$ Department of Mini-invasive Spinal Surgery, Third Hospital of Henan Province, Zhengzhou, Henan, China \\ ${ }^{*}$ Equal contribution
}

Correspondence to: Zhipeng Dai; email: daizp@hust.edu.cn

Keywords: osteonecrosis of the femoral head, mesenchymal stem cell, m6A, METTL14, Wnt signaling pathway

Received: June 18, $2021 \quad$ Accepted: November 22, $2021 \quad$ Published: December 15, 2021

Copyright: (C) 2021 Cheng et al. This is an open access article distributed under the terms of the Creative Commons Attribution License (CC BY 3.0), which permits unrestricted use, distribution, and reproduction in any medium, provided the original author and source are credited.

\begin{abstract}
Imbalanced osteogenic/adipogenic differentiation of bone marrow mesenchymal stem cells (BMSCs) is considered the core pathological characteristic of steroid-associated osteonecrosis of the femoral head (SONFH). N6-Methyladenosine (m6A) is the most common type of RNA modification in eukaryotic cells and participates in various physiological and pathological processes. However, the relationship between m6A modification and SONFH has not been reported. In the present study, we aimed to explore the roles of m6A modifications and methyltransferase METTL14 in SONFH. Our results showed that the m6A levels were downregulated in femoral head tissues and BMSCs from SONFH patients, and this effect was attributed to the reduction of METTL14. Furthermore, METTL14 overexpression in BMSCs from SONFH patients enhanced cell proliferation and osteogenic differentiation. We further identified PTPN6 as the downstream target of METTL14 by mRNA sequencing. Mechanistically, METTL14 regulated PTPN6 expression by increasing PTPN6 mRNA stability in an m6A-dependent manner. Moreover, PTPN6 knockdown abrogated the beneficial effects of METTL14 overexpression on BMSCs. Additionally, we found that METTL14 activated the Wnt signaling pathway, and this effect was caused by the interaction of PTPN6 and GSK-3 $\beta$. In conclusion, we elucidated the functional roles of METTL14 and m6A methylation in SONFH BMSCs and identified a novel RNA regulatory mechanism, providing a potential therapeutic target for SONFH.
\end{abstract}

\section{INTRODUCTION}

Steroid-associated osteonecrosis of the femoral head (SONFH) is a severe clinical complication caused by the use of glucocorticoids. Studies have confirmed that the occurrence and progression of SONFH are related to the course and dose of glucocorticoids [1]. With the increasing incidence of SONFH, the molecular mechanism urgently needs to be identified.

Bone marrow mesenchymal stem cells (BMSCs) are pluripotent stem cells with self-renewal and multidirectional differentiation potential and can differentiate into osteoblasts, adipocytes, endotheliocytes and chondrocytes [2-5]. Thus, BMSCs play a crucial role in bone metabolism, tissue repair and are considered ideal seed cells for tissue engineering [6-8]. Moreover, these cells are also closely related to the development of bone disorders. Recent studies have demonstrated that the progression of SONFH is associated with BMSCs dysfunction, which is mainly manifested by the defection of osteogenic differentiation and the enhancement of adipogenic differentiation [9-11]. In previous studies, BMSCs in proximal femoral bone marrow were significantly reduced in the rabbit model of SONFH, and BMSCs 
from SONFH patients had decreased proliferation and osteogenic activity [12-14]. Therefore, strategies promoting the BMSC proliferation and osteogenic ability may be a promising therapeutic method for SONFH.

RNA methylation accounts for more than $60 \%$ of RNA modifications [15]. Among them, m6A is the most common RNA methylation modification in eukaryotic cells [16]. In mammalian cells, the m6A modification is a reversible process regulated by $\mathrm{m} 6 \mathrm{~A}$ writers, erasers and readers [17]. The formation of m6A methylation is catalyzed by the methyltransferase complex that consists of m6A writers, among which METTL3, METTL14 and WTAP play vital roles [18]. Conversely, ALKBH5 and FTO act as erasers to reverse the m6A modification [19]. The readers consist of YTH domain proteins and the nuclear heterogeneous protein family, which are responsible for reading the methylation information and participating in RNA translation and degradation [20]. Previous studies have demonstrated that m6A modification involves in the life cycle of RNA, affecting the RNA stability, translation efficiency, alternative splicing and localization [21-24]. Aberrant levels of m6A have been confirmed to contribute to a variety of diseases, such as tumors, neurological diseases, and metabolic diseases [25-27], and m6A RNA methylation also regulates the fate of BMSCs [28] and involved in the progression of diseases related to BMSCs abnormalities. For instance, Liu et al. discovered that METTL3 promoted BMSCs osteogenesis by mediating m6A methylation of BMP2 transcripts and supposed that METTL3 could mitigate ovariectomy-induced osteoporosis [29]. Xie et al. revealed that TNF- $\alpha$ induced $\mathrm{m} 6 \mathrm{~A}$ modification in ELMO1 3'UTR triggers directional migration of mesenchymal stem cell in ankylosing spondylitis patients [30]. Wang et al. elucidated a new pathogenesis of osteoporosis, that is, FTO mediates Runx 2 mRNA demethylation to inhibit the osteogenic differentiation of BMSCs [31]. However, the roles of m6A modification and the underlying regulatory mechanisms in SONFH remain unclear.

PTPN6 belongs to the protein tyrosine phosphatase family, which is mainly expressed in hematopoietic stem cells. PTPN6 regulates receptor tyrosine kinases by binding to target proteins and dephosphorylating phosphorylated tyrosine substrates, which play a significant role in cell differentiation, proliferation, and biological functions [32]. Previous studies have confirmed that PTPN6 is involved in the molecular mechanisms of the progression of various disease [33, 34]. However, the underlying mechanism by which PTPN6 is regulated, especially via m6A modification, and the roles of PTPN6 in SONFH are still unknown.
Here, we found that the levels of m6A modification and METTL14 expression in SONFH tissues and BMSCs were significantly lower than those in normal tissues and cells. Overexpression of METTL14 in SONFH BMSCs significantly enhanced cell proliferation and osteogenic differentiation, and regulated the expression of lineage-specific transcription factors including RUNX2, PPAR $\gamma$ and C/EBPs. Molecular mechanism analysis indicated that METTL14 mediated the m6A modification and stability of PTPN6 mRNA, which further up-regulated PTPN6 expression in SONFH BMSCs. Rescue experiments showed that PTPN6 knockdown abrogated the effects of METTL14 on cell proliferation and differentiation. Furthermore, we demonstrated that PTPN6-mediated METTL14 activated the Wnt signaling pathway by interacting with GSK-3 $\beta$. Our study laid the foundation for future research on the roles of m6A modification in SONFH, and provided a novel target for the treatment of SONFH.

\section{RESULTS}

\section{Identification of BMSCs}

When the cells were passaged to the second generation, they had a typical long fusiform appearance under the microscope (Figure 1A). Besides, the cells have the potential to differentiate into osteoblasts and adipocytes (Figure 1B, 1C). Furthermore, we analyzed four known surface markers associated with human bone marrow mesenchymal stem cells by flow cytometry. The results showed that the cells were positive for CD29 and CD44, but negative for CD34 and CD45 (Figure 1D). These results indicated that the isolated cells were human BMSCs.

\section{METTL14 was responsible for the aberrant $\mathbf{m 6 A}$ modifications in BMSCs from SONFH patients}

To explore the potential role of m6A modification in SONFH, we initially examined the level of m6A in the total RNA of femoral head tissues and BMSCs. As shown in Figure 2A, the m6A content in SONFH tissues and BMSCs was significantly lower than that in normal group. According to the previous studies, m6A modifications are catalyzed by $\mathrm{m} 6 \mathrm{~A}$ methyltransferase and m6A demethylase, so we next examined the mRNA levels of m6A modification associated genes, including METTL3, METTL14, WTAP, FTO and ALKBH5. Interestingly, the m6A methyltransferase METTL14 was obviously down-regulated in osteonecrosis tissues and BMSCs from SONFH patients, while the demethylase ALKBH5 was up-regulated in SONFH tissues, but not in SONFH BMSCs. The levels of METTL3, WTAP and FTO exhibited no significant 
differences between the two groups (Figure 2B). Thus, we hypothesized that METTL14 was a critical regulator that contributed to the abnormal m6A modifications. We further performed IHC analysis with femoral head tissues, and found that the positive rate of METTL14 in necrotic femoral head tissues was significantly lower than that in healthy femoral head tissues (Figure 2C). Moreover, western blotting indicated that protein levels of METTL14 were down-regulated in BMSCs from SONFH patients compared to those in normal BMSCs (Figure 2D). To further verify the effect of METTL14 on the m6A modification in BMSCs, we overexpressed METTL14 in SONFH BMSCs, and qRT-PCR and western blotting were used to determine the transfection effect (Figure 2E, 2F). Then, we detected the m6A content in cells. The results showed that the m6A level was increased significantly upon METTL14 overexpression (Figure 2G). Together, these data demonstrated that the m6A level was decreased in SONFH femoral head tissues and BMSCs, and that METTL14 was the regulator involving the abnormal m6A modification.

\section{METTL14 up-regulation promoted the proliferation and osteogenesis of SONFH BMSCs}

To investigate the biological functions of METTL14, we assessed the proliferation and osteogenesis abilities in SONFH BMSCs transfected with OE-METTL14. The results of CCK-8 and EdU assays confirmed that METTL14 overexpression significantly promoted the proliferation capacity of BMSCs (Figure 3A, 3B). Furthermore, METTL14 up-regulation augmented cell ALP activity (Figure 3C). Alizarin red staining and oil
A Cell morphology

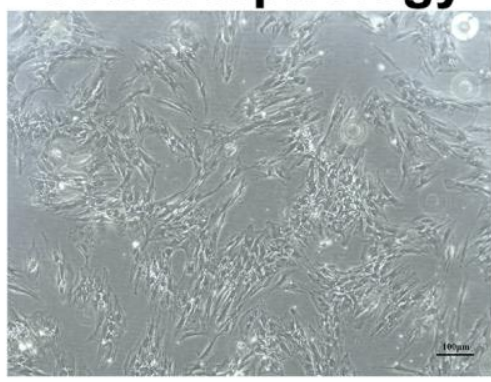

B Osteogenesis

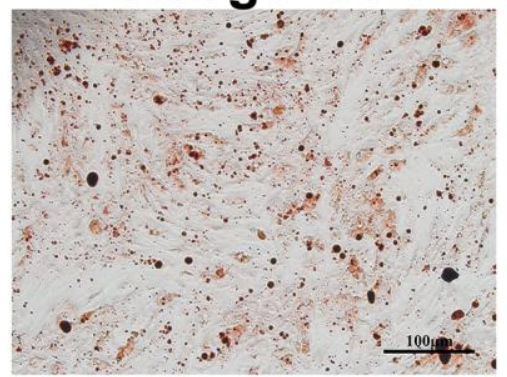

C Adipogenesis

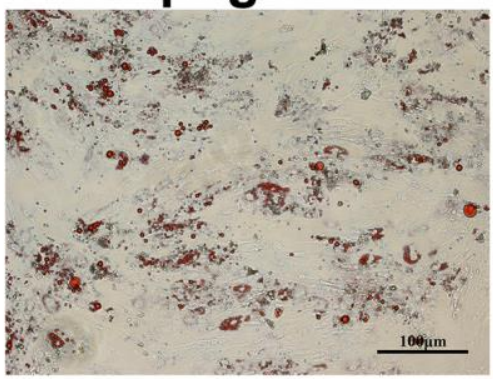

CD29

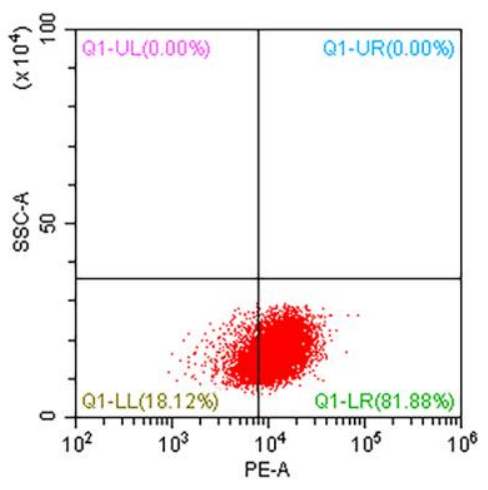

CD34

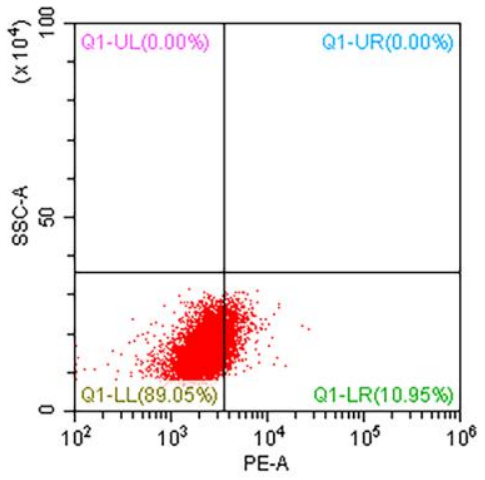

CD44

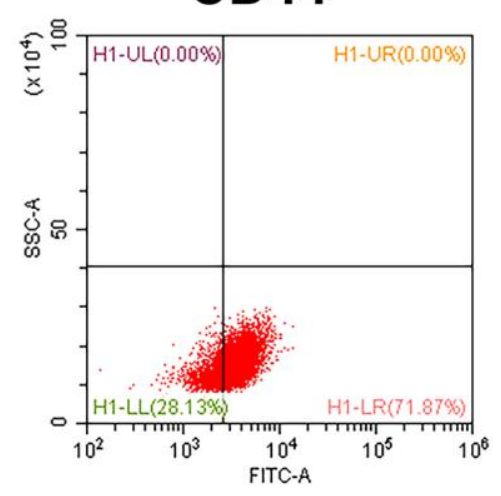

CD45

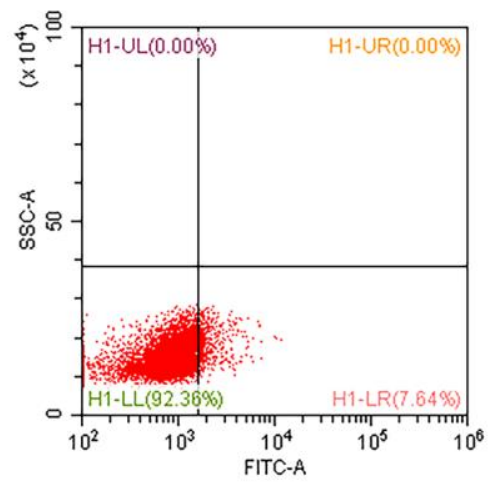

Figure 1. Identification of BMSCs. (A) The appearance of BMSCs cultured to the second generation. Scale bar $=100 \mu \mathrm{m}$. $(\mathbf{B})$ The osteogenesis differentiation of BMSCs was confirmed by alizarin red staining. Scale bar $=100 \mu \mathrm{m}$. (C) The potential of BMSCs to differentiate into adipocytes was verified by oil red staining. Scale bar $=100 \mu \mathrm{m}$. (D) The surface markers of BMSCs were identified by flow cytometry. 
red staining showed that the ability to differentiate into osteoblasts was enhanced and the potential to differentiate into adipocytes was decreased when METTL14 expression was overexpressed (Figure 3D). Western blotting results demonstrated that with METTL14 overexpression, the levels of the osteogenic- related gene RUNX2 was up-regulated, while the expression of adipogenic-related genes PPAR $\gamma, \mathrm{C} / \mathrm{EBP} \alpha$ and $\mathrm{C} / \mathrm{EBP} \beta$ were down-regulated in SONFH BMSCs (Figure 3E). Taken together, these results suggested that METTL14 promotes the proliferation and osteogenesis of BMSCs from SONFH patients.
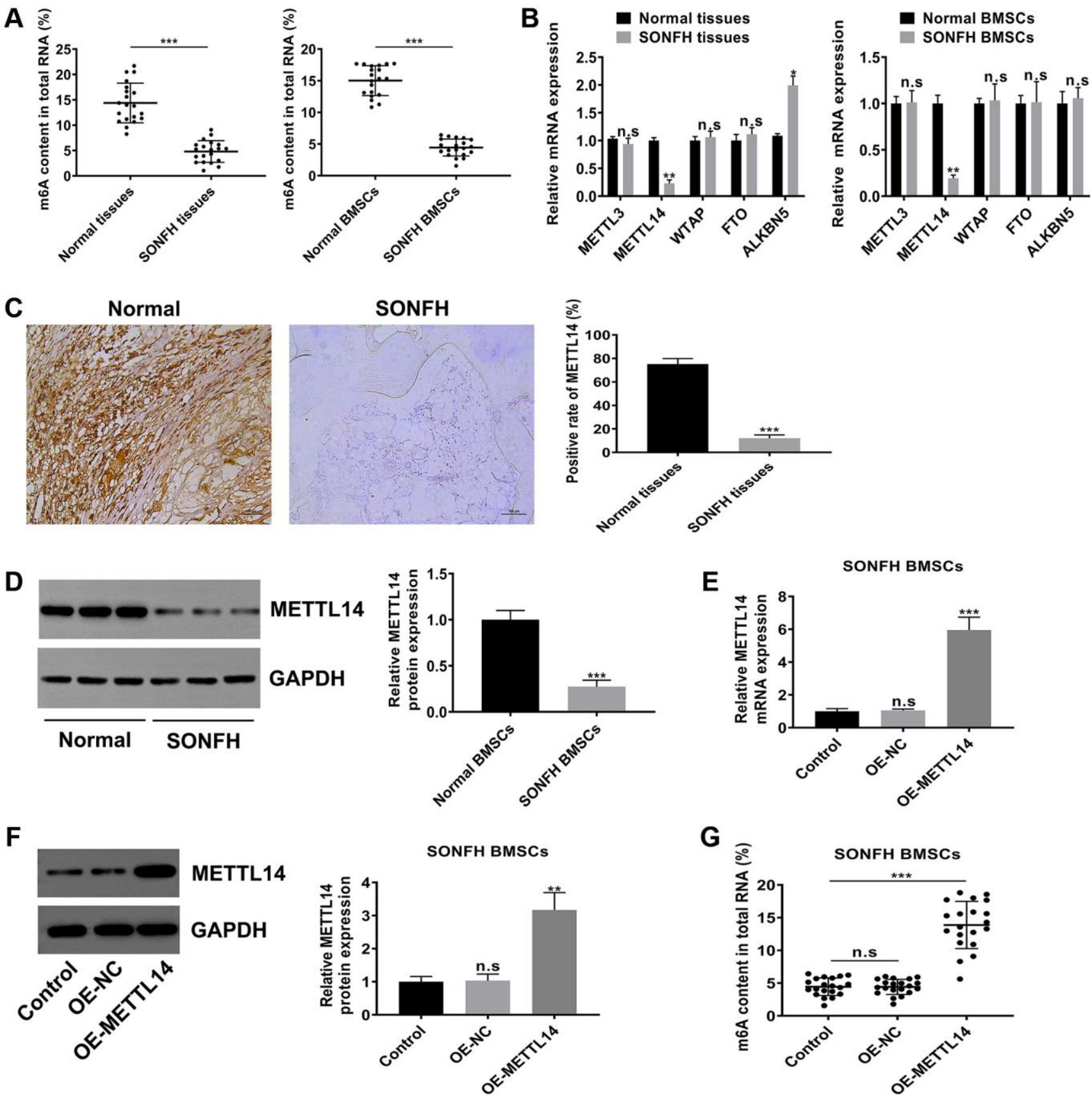

Figure 2. METTL14 was responsible for the aberrant m6A modification in BMSCs from SONFH patients. (A) The m6A content in total RNA of SONFH tissues and BMSCs $(n=20)$ and normal tissues and BMSCs $(n=20)$. (B) mRNA levels of m6A modification associated genes in SONFH tissues and BMSCs and normal tissues and BMSCs. (C) IHC assay determined the expression of METTL14 in osteonecrosis tissues and normal tissues. Scale bar $=100 \mu \mathrm{m}$. (D) The protein levels of METTL14 in SONFH BMSCs and normal BMSCs were measured by western blot. (E, F) The efficiency of METTL14 overexpression in SONFH BMSCs was confirmed by qRT-PCR and western blot analysis. (G) Quantitative m6A methylation assay was used to define the effect of METTL14 overexpression on the level of m6A modification in BMSCs from SONFH patients. ${ }^{*} P<0.05,{ }^{* *} P<0.01,{ }^{* * *} P<0.001$, n.s is no significance. 
METTL14-dependent m6A modifications regulated the expression of PTPN6

M6A modification mostly exists in mRNA and assumes as a form of posttranscriptional regulation, which further affects the expression of the modified gene. To identify the downstream target of METTL14, we performed mRNA sequencing on BMSCs from SONFH patients transfected with OE-METTL14 or OE-NC. Differentially expressed genes were screened based on $p$-value $<0.05$ and false discovery rate $<0.05$. Compared to control cells, METTL14-overexpressing cells had 101 genes with increased expression and 142 genes were decreased, and these genes were clustered
A

A

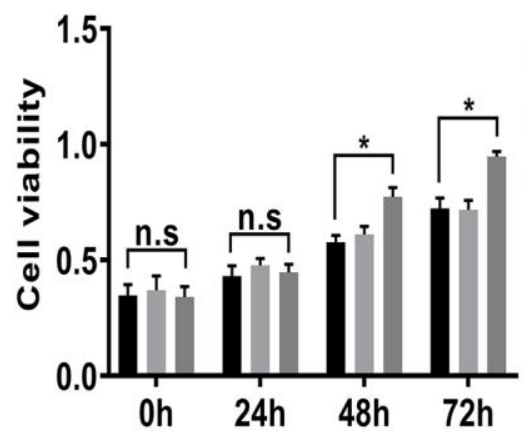

C

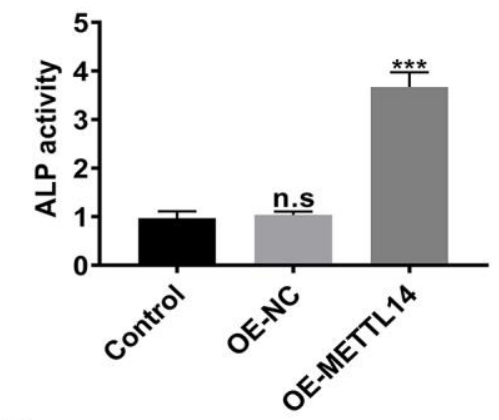

E

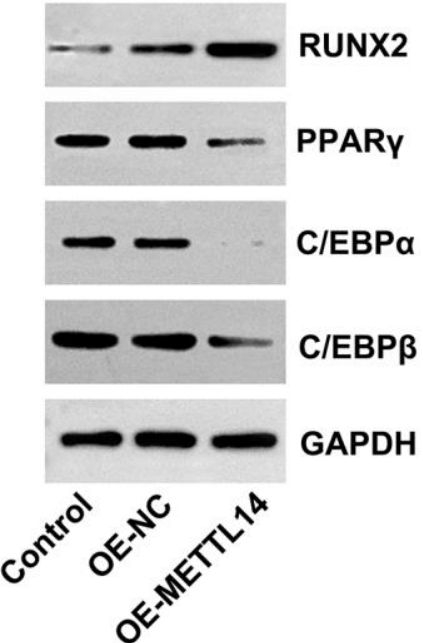

B
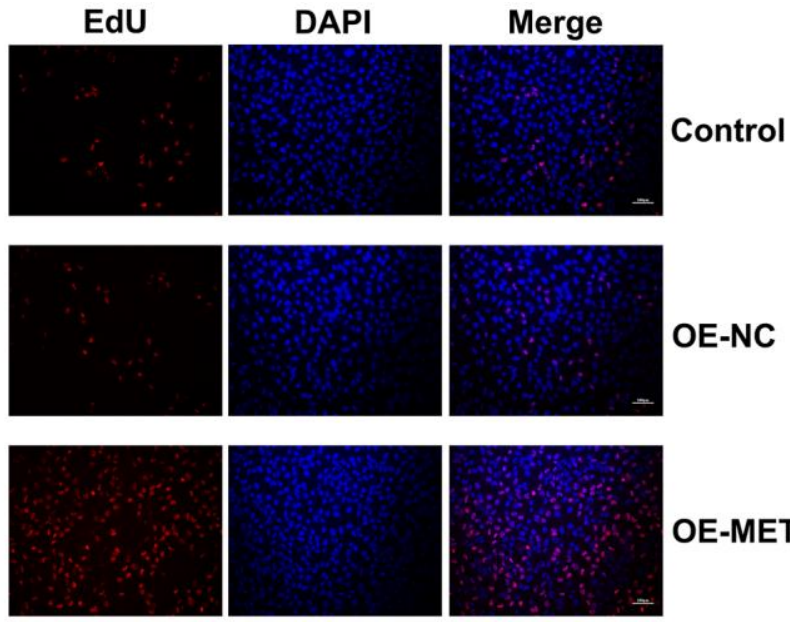

OE-NC

D
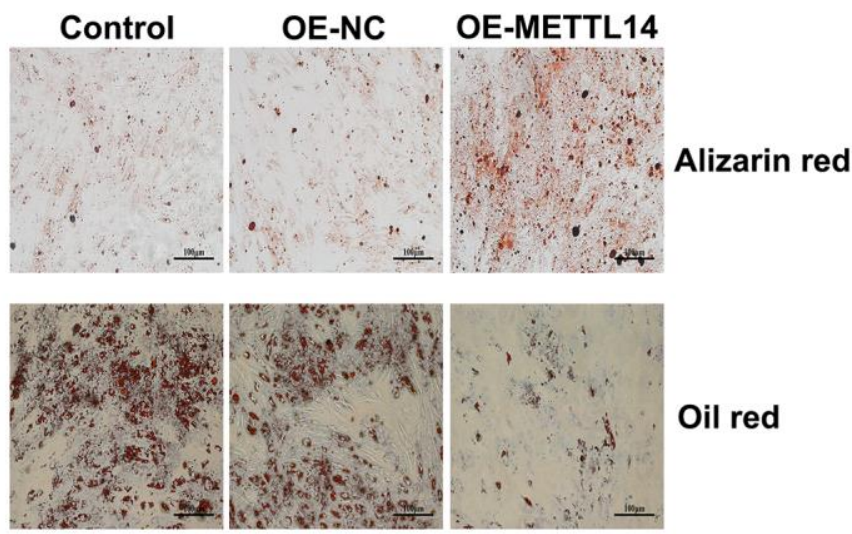

Oil red

\section{OE-METTL14}

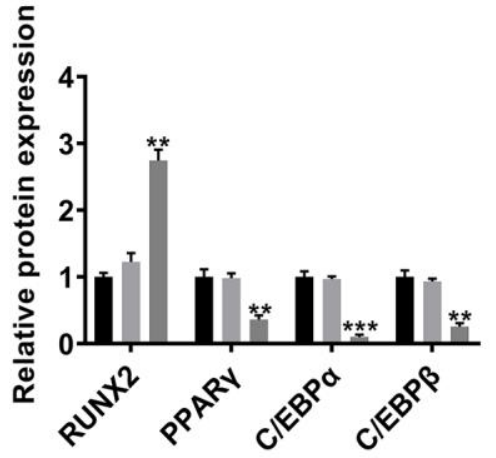

Control

OE-NC

OE-METTL14

Figure 3. METTL14 up-regulation promoted the proliferation and osteogenesis of SONFH BMSCs. (A) The effect of METTL14 upregulation on the cell viability of SONFH BMSCs was analyzed by CCK-8 assay. (B) EdU assay was used to detect the influence of METTL14 overexpression on the proliferation of SONFH BMSCs. Scale bar $=100 \mu \mathrm{m}$. (C) The ALP activity assay provided evidence that METTL14 upregulation enhanced the ability of osteogenic differentiation of BMSCs from SONFH patients. (D) METTL14 overexpression promoted the ability of osteogenic differentiation and suppressed the adipogenesis capacity of SONFH BMSCs were confirmed by alizarin red staining and oil red staining. Scale bar $=100 \mu \mathrm{m}$. (E) The expression of osteogenic-related and adipogenic-related genes in BMSCs with METTL14 overexpression were analyzed by western blot. ${ }^{*} P<0.05,{ }^{* *} P<0.01,{ }^{* * *} P<0.001$, n.s is no significance. 
analyzed and presented as a heat map (Figure 4A). Among differentially expressed genes, the expression of PTPN6 was most significantly affected by METTL14 (fold change was the highest). The data of qRT-PCR displayed that PTPN6 mRNA levels in osteonecrosis tissues was decreased compared with control femoral
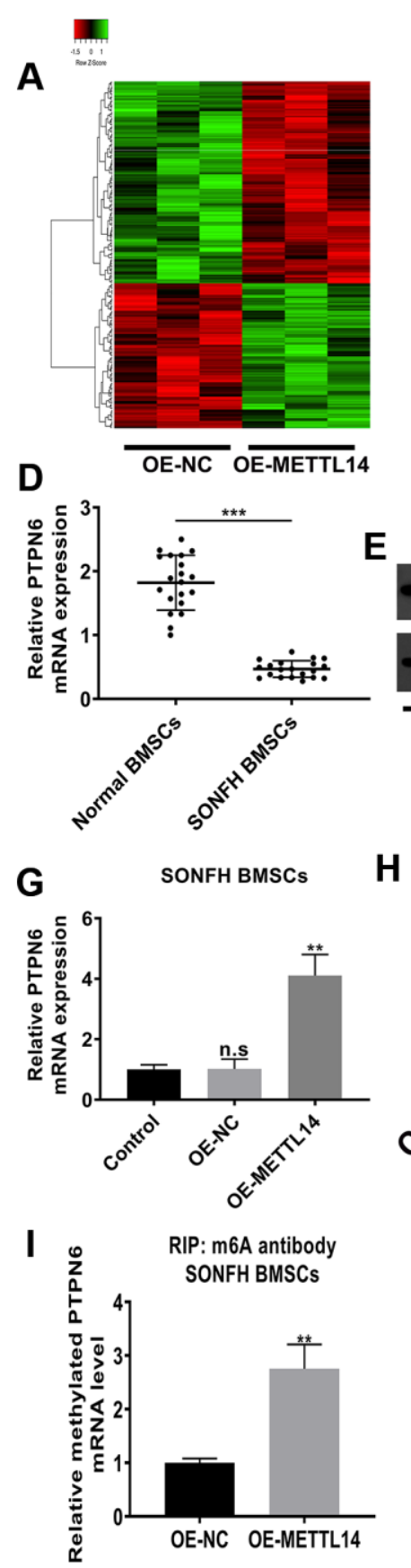

B

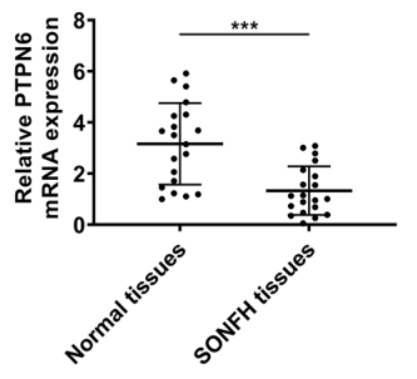

$\mathbf{E}$

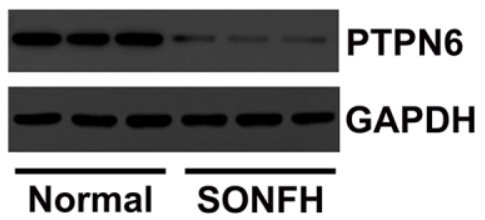

H

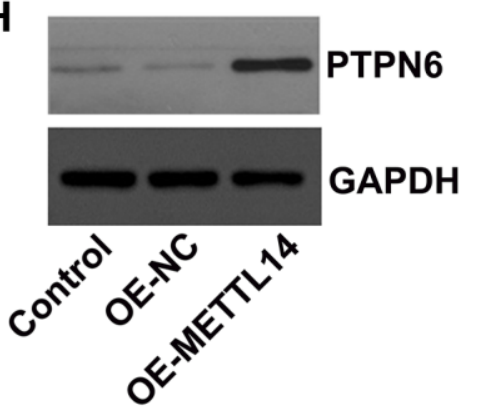

C

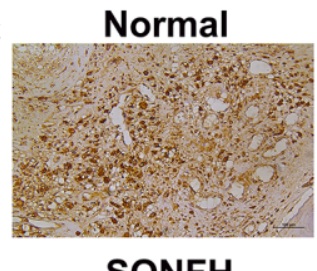

SONFH
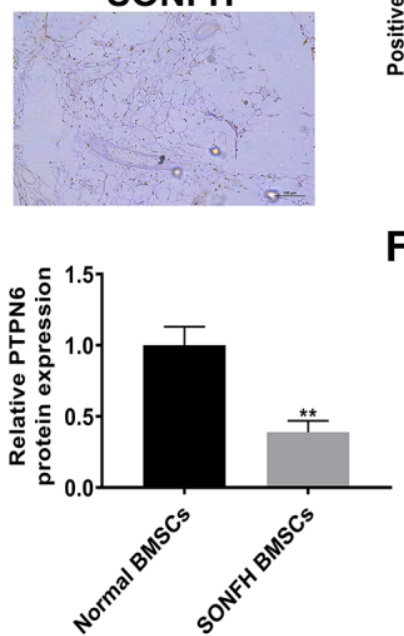

SONFH BMSCs
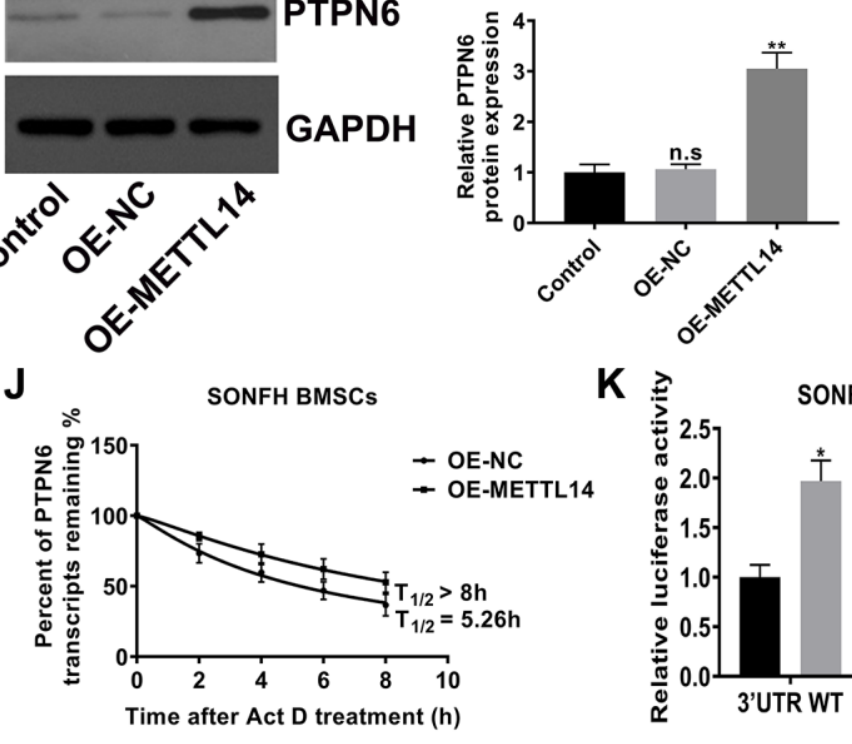
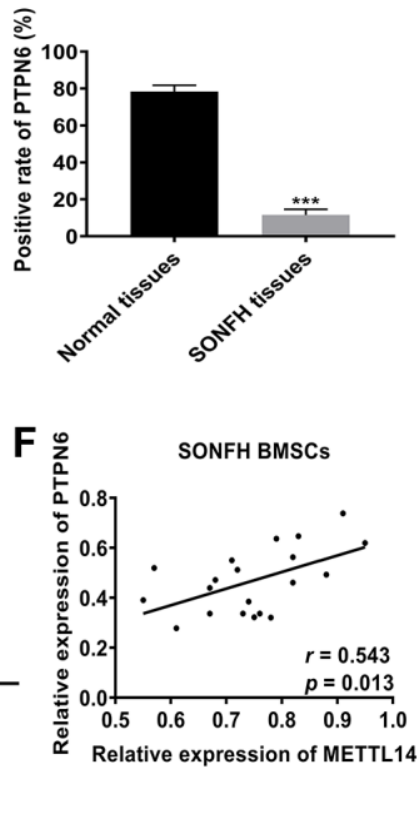

(

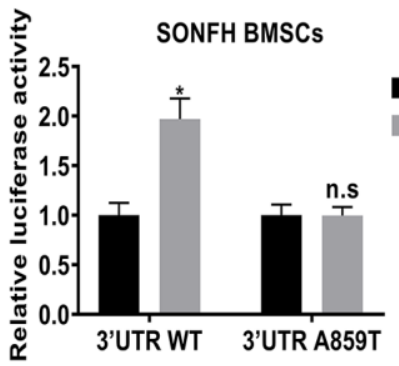

OE-NC

OE-METTL14

Figure 4. METTL14-dependent m6A modification regulated the expression of PTPN6. (A) Heat map of normalized gene expression levels of SONFH BMSCs transfected with OE-NC or OE-METTL14. Red indicates down-regulation of gene expression and Green indicates up-regulation of gene expression. (B) PTPN6 mRNA levels in normal tissues $(n=20)$ and osteonecrosis tissues ( $n=20$ ) were measured by qRT-PCR. (C) IHC assay detected the expression of PTPN6 in femoral head tissues of two group. Scale bar = $100 \mu \mathrm{m}$. (D, E) qRTPCR and western blot quantified the abundance of PTPN6 in normal BMSCs and SONFH BMSCs. (F) The correlation between METTL14 and PTPN6 expression in SONFH BMSCs was analyzed by Pearson's Correlation Coefficient. (G, H) qRT-PCR and western blot confirmed that PTPN6 expression increased with the up-regulation of METTL14 in SONFH BMSCs. (I) The methylated PTPN6 mRNA level in the METTL14 overexpressed BMSCs was determined by MeRIP-qPCR. (J) The mRNA half-life of PTPN6 in SONFH BMSCs transfected with OE-NC or OEMETTL14. (K) SONFH BMSCs were pre-transfected with wild-type or mutated PTPN6-3'UTR plasmids, and then treated with OE-NC or OEMETTL14. The luciferase activity was normalized to firefly luciferase activity. ${ }^{*} P<0.05,{ }^{* *} P<0.01,{ }^{* * *} P<0.001$, n.s is no significance. 
head tissues (Figure 4B). IHC assay also provided consistent results, the positive staining of PTPN6 in necrotic femoral head tissues was significantly less than that in normal tissues (Figure 4C). Moreover, the qRT-PCR and western blot results indicated that the level of PTPN6 in BMSCs of SONFH was significantly lower than that in normal BMSCs (Figure 4D, 4E), and PTPN6 mRNA expression was positively correlated with METTL14 mRNA in SONFH BMSCs (Figure 4F). Besides, in SONFH BMSCs, the mRNA and protein levels of PTPN6 were increased with METTL14 up-regulation (Figure $4 \mathrm{G}, 4 \mathrm{H})$. We then investigated the m6A modification status of PTPN6 by performing MeRIP-qPCR assay. The results validated that the up-regulation of METTL14 enriched the m6A modification level of PTPN6 mRNA (Figure 4I). Furthermore, we carried out the RNA stability assay to analyze the half-life of PTPN6 mRNA. As shown in Figure 4J, the half-life of PTPN6 mRNA was 5.26 hours in SONFH BMSCs, while the half-life of PTPN6 was longer than 8 hours in METTL14 overexpression cells. To further elucidate the mechanism of METTL14 regulation of PTPN6 mRNA stability, we performed the luciferase reporter assay and found that METTL14 overexpression reinforced the activity of the luciferase construct containing the wild type 3'UTR of PTPN6 mRNA. Mutation at position 859 (A to T) almost completely rendered resistance to the luciferase activity of METTL14 overexpression (Figure 4K). Collectively, METTL14-dependent m6A modification regulates the expression of PTPN6 by enhancing the stability of PTPN6 mRNA.

\section{PTPN6 knockdown abrogated the beneficial effects of METTL14 overexpression on SONFH BMSCs}

To explore whether PTPN6 is critical to the effects of METTL14 on SONFH BMSCs, we silenced PTPN6 in METTL14 overexpression cells. The efficiency of PTPN6 knockdown was confirmed by qRT-PCR and western blot assays (Figure 5A, 5B). The CCK-8 assay and EdU assay indicated that silenced PTPN6 dramatically abrogated the effect of METTL14 on cell proliferation (Figure 5C, 5D). The results of alizarin red staining, oil red staining and ALP activity assays demonstrated that PTPN6 knockdown reversed the promotion of METTL14 overexpression on the osteogenic differentiation of BMSCs (Figure 5E, 5F). In addition, when we weakened PTPN6 expression in METTL14-overexpressing cells, the protein level of RUNX2 was down-regulated, and those of PPAR $\gamma$, $\mathrm{C} / \mathrm{EBP} \alpha$ and $\mathrm{C} / \mathrm{EBP} \beta$ were up-regulated (Figure 5G). Overall, these results suggested that PTPN6 is decisive to the beneficial effect of METTL14 on BMSCs from SONFH patients.

\section{PTPN6-mediated METTL14 activated the Wnt signaling pathway}

The canonical Wnt signaling pathway is the essential signal transduction involved in the osteogenic differentiation of stem cells [35-37]. To verify whether METTL14 affects the activity of the Wnt signaling pathway in SONFH BMSCs, we initially conducted TOP/FOP-Flash reporter assay. The results showed that the activity of the Wnt signaling pathway was heightened in METTL14 overexpressed cells, and PTPN6 knockdown reversed this effect (Figure 6A). The western blot indicated that METTL14 overexpression resulted in the up-regulation of total and nuclear $\beta$-catenin and the decrease of phosphorylated $\beta$ catenin, while PTPN6 knockdown diminished the abundance of $\beta$-catenin in total protein and nucleoprotein, and the phosphorylation of $\beta$-catenin protein was augmented simultaneously (Figure 6B, 6C). Moreover, the immunofluorescence staining showed lower level of $\beta$-catenin in the nuclei of the control group than METTL14 overexpression group. The upregulation of METTL14 obviously induced the translocation of $\beta$-catenin (red) into the nucleus (blue). PTPN6 knockdown evidently attenuated the effect of METTL14, as PTPN6 knockdown almost completely blocked the nuclear translocation of $\beta$-catenin (Figure 6D). GSK-3 $\beta$ assumes an essential role in the degradation of $\beta$-catenin. According to co-IP experiment, we found that PTPN6 could interact with GSK-3 $\beta$ in BMSC (Figure 6E). In addition, in SONFH BMSCs, PTPN6 inhibition obviously reduced the phosphorylation level of GSK-3 $\beta$ (Figure 6F). In conclusion, PTPN6-mediated METTL14 activates the Wnt signaling pathway in BMSCs from SONFH patients.

\section{DISCUSSION}

Glucocorticoids are widely used to clinically treat autoimmune diseases and inflammatory-dependent diseases. Therefore, the incidence of femoral head necrosis caused by high-dose glucocorticoids is gradually increasing. With the in-depth study of the pathogenesis of SONFH, the role of BMSCs has attracted wide attention [38]. The previous study found that in the animal model of SONFH, there was a large amount of lipid deposition in the proximal femur and femoral head [13], and the osteogenic ability of BMSCs was inhibited $[12,14]$, which suggested that the bone repair defect caused by imbalanced osteogenic/adipogenic differentiation of BMSCs may be an important characteristic of SONFH.

m6A RNA modification is the most abundant endogenous RNA modification pattern, which widely 
exists in mRNA and non-coding RNA in eukaryotic cells [21]. Recent studies have found that m6A modification is dynamic and reversible in different developmental stages, tissues and physiological processes [19, 39]. Similar to DNA methylation or histone methylation, m6A RNA methylation is also catalyzed by methyltransferase and demethylase systems [17]. As the main component of the m6A methyltransferase complex, METTL14 is involved in the progression of multiple diseases in an m6A dependent manner. Weng et al. demonstrated that METTL14 inhibits hematopoietic progenitor
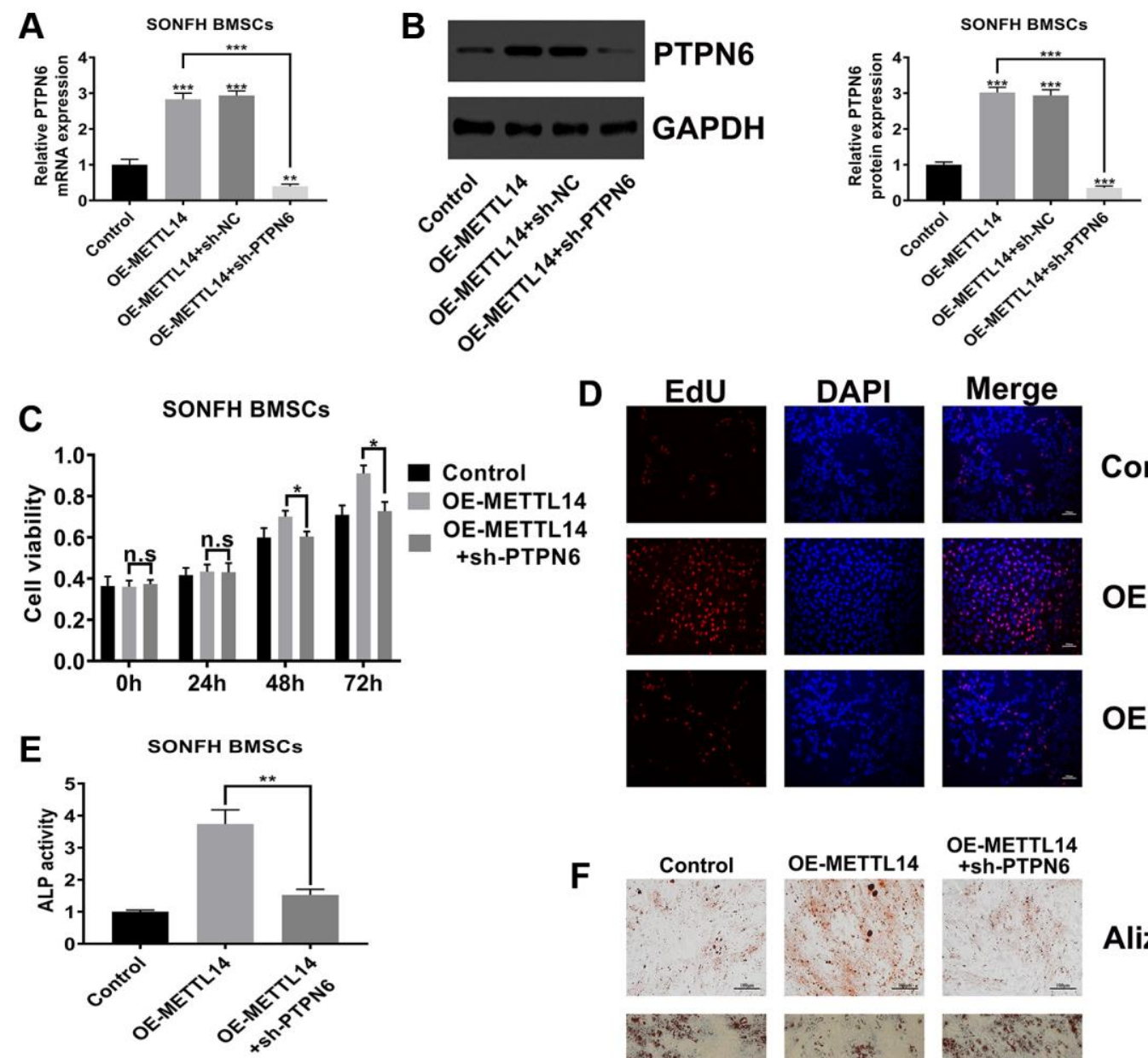

Control

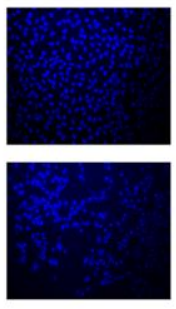

OE-METTL14

G

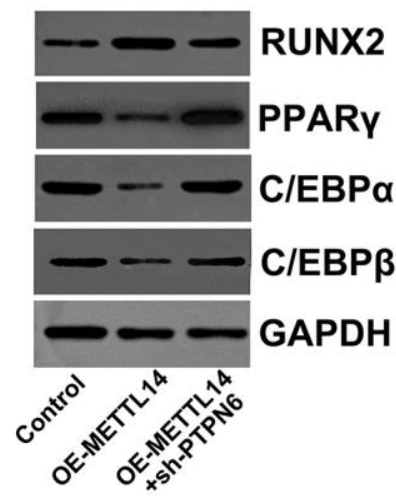

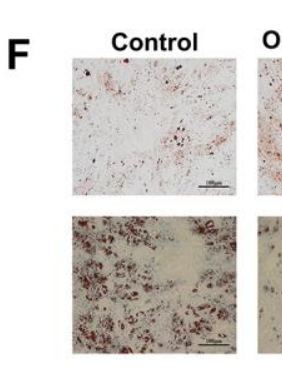

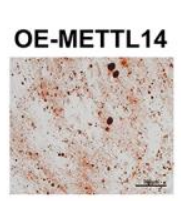

\section{OE-METTL14}

+sh-PTPN6
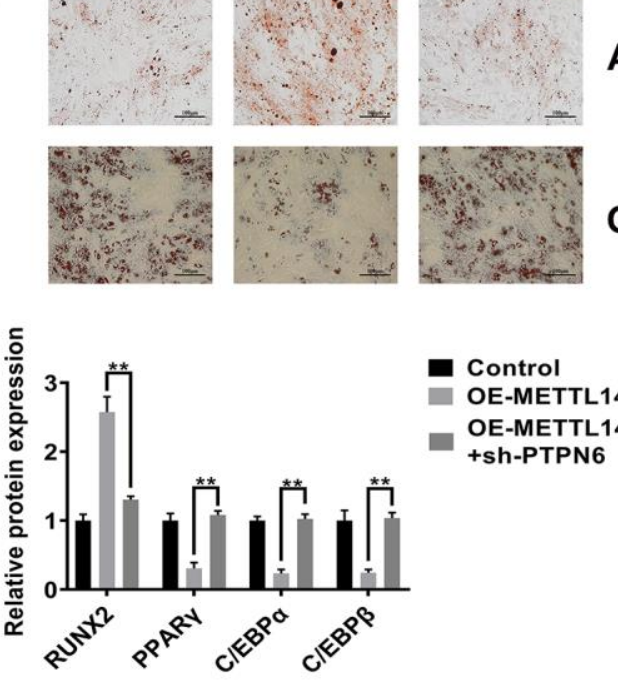

Alizarin red

OE-METTL14+sh-PTPN6

\section{Oil red}

Figure 5. PTPN6 knockdown abrogated the beneficial effects of METTL14 overexpression on SONFH BMSCs. (A, B) qRT-PCR and western blot validated the efficiency of PTPN6 knockdown in METTL14 overexpression cells. (C, D) The CCK-8 and EdU assay evaluated the reversal effect of PTPN6 knockdown on cell proliferation induced by METTL14 overexpression. Scale bar $=100 \mu \mathrm{m}$. (E) The ALP activity assay was used to assess the reversal effect of PTPN6 knockdown on the osteogenesis promoted by METTL14 up-regulation. (F) The effects of sh-PTPN6 on the abilities of osteogenic differentiation and adipogenic differentiation of METTL14 overexpression cells were analyzed by Alizarin red staining and oil red staining. Scale bar $=100 \mu \mathrm{m}$. (G) Knockdown of PTPN6 counteracted the regulatory effect of METTL14 overexpression on osteogenic and adipogenic related genes. ${ }^{*} P<0.05,{ }^{* *} P<0.01,{ }^{* * *} P<0.001$, n.s is no significance. 
differentiation and promotes leukemogenesis via m6A modification [40]. Ma et al. revealed that METTL14 suppresses the metastatic potential of hepatocellular carcinoma by modulating primary microRNA-126 process in m6A dependent manner [41]. However, the role of m6A modification in SONFH has not been reported. In this study, we found that the expression level of METTL14 was down-regulated in necrotic bone tissues and SONFH BMSCs, which resulted in a decrease of the m6A level. Moreover, overexpression of METTL14 in BMSCs from SONFH patients not only up-regulated the level of m6A modification in total RNA, but also enhanced cell proliferation and osteogenic differentiation, and simultaneously inhibited adipogenic differentiation.

PTPN6 is a protein tyrosine phosphatase located in the cytoplasm and participates in the regulation of immune
A

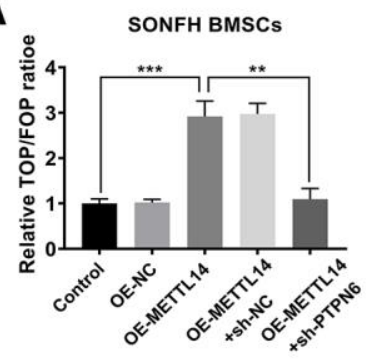

C

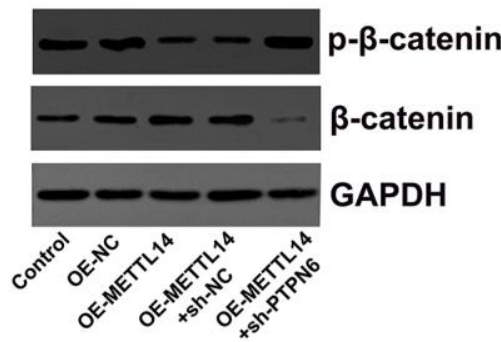

B
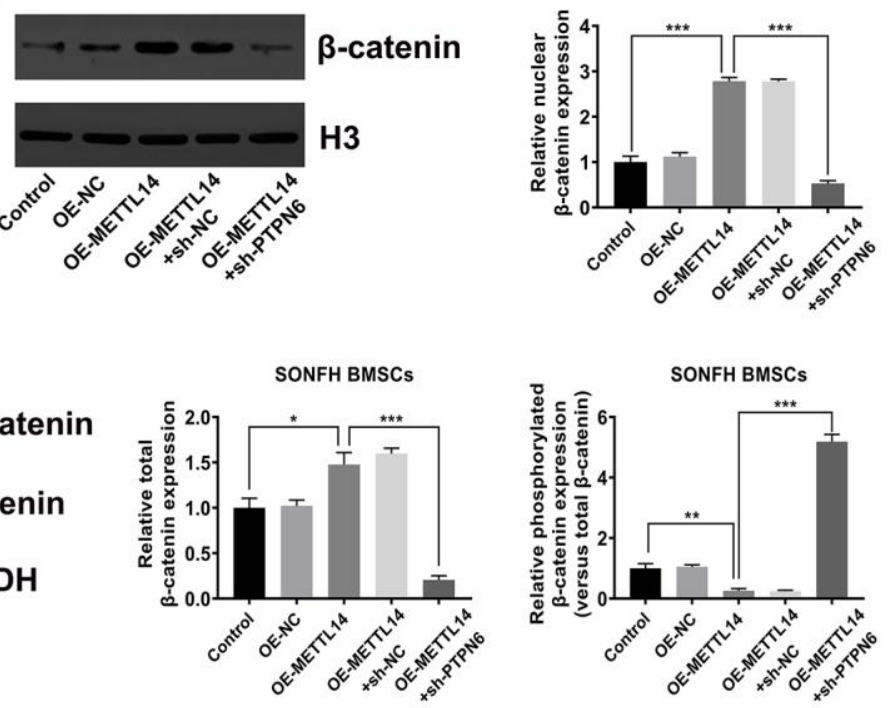
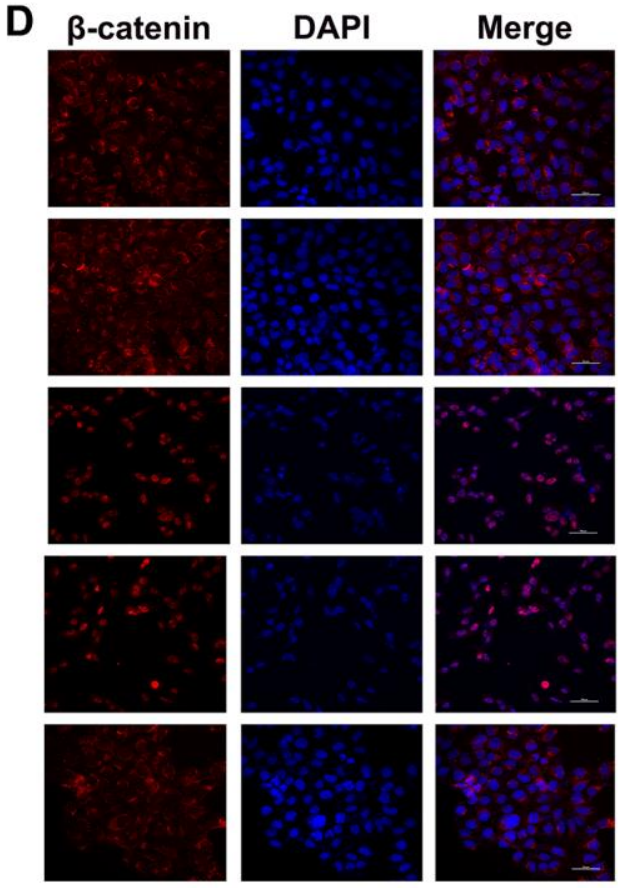
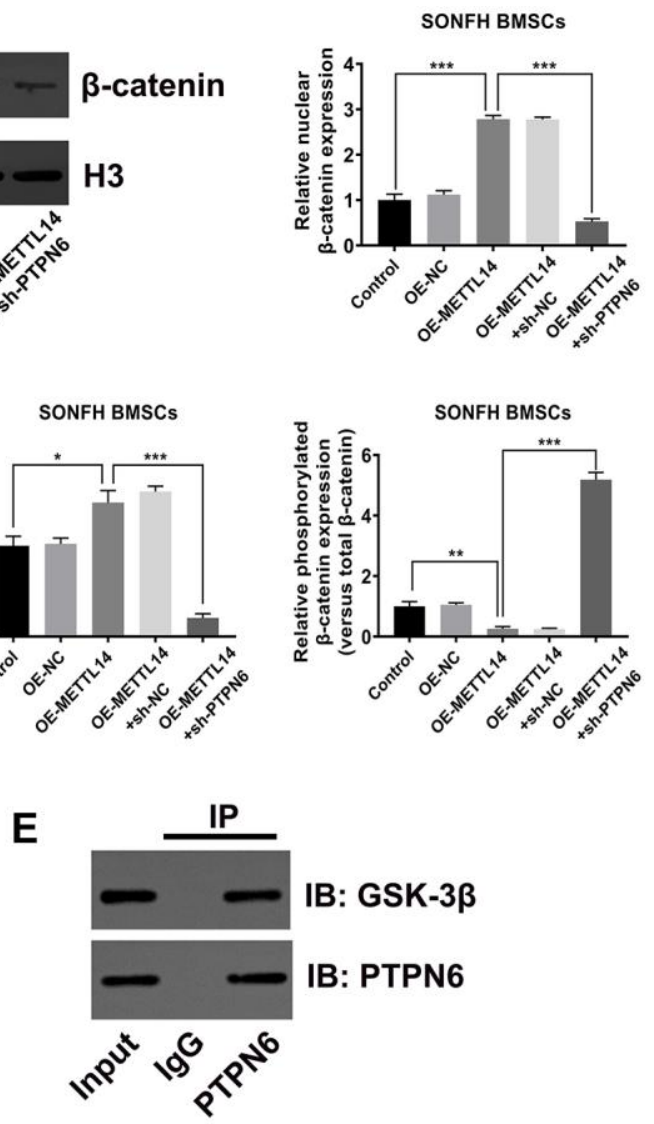

\section{IB: GSK-3 $\beta$}

IB: PTPN6

$\mathbf{F}$

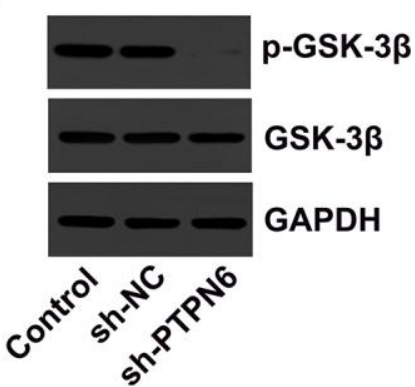

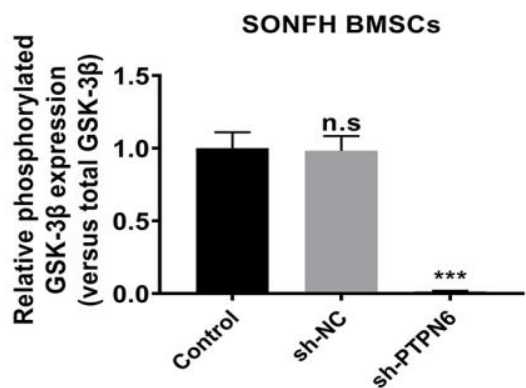

Figure 6. PTPN6-mediated METTL14 activated the Wnt signaling pathway. (A) The activity of Wnt signaling in SONFH BMSCs was analyzed by TOP/FOP-Flash reporter assay. (B) The protein level of $\beta$-catenin in the nucleus was measured by western blot. (C) Western blot detected the total and phosphorylated $\beta$-catenin levels in SONFH BMSCs treated as indicated. (D) Immunofluorescence staining was used to analyze the distribution of $\beta$-catenin in the cytoplasm and nucleus after METTL14 overexpression and PTPN6 knockdown. Scale bar $=50 \mu \mathrm{m}$. (E) The interaction of PTPN6 and GSK-3 $\beta$ in BMSCs was verified by co-IP assay. (F) The effect of PTPN6 inhibition on the phosphorylation level of GSK-3 $\beta$ in SONFH BMSCs was determined by western blot. ${ }^{*} P<0.05,{ }^{* *} P<0.01,{ }^{* * *} P<0.001$, n.s is no significance. 
function, tissue inflammation, cell proliferation and other various pathological processes [42-44]. Jiang et al. found that SHP1 (alias of PTPN6) knockout mice were susceptible to osteoporosis due to SHP1 could bind to GSK-3 $\beta$ and inhibit its kinase activity, thus activating the Wnt signaling pathway [45]. As an evolutionarily conserved signaling pathway, the canonical Wnt signaling pathway is crucial for renewal, proliferation, and differentiation of stem cells during bone development and tissue homeostasis [46, 47]. When the Wnt receptor complex is activated, GSK-3 $\beta$ is triggered to dissociate from the APC/Axin/GSK-3 $\beta$ complex, and the stable $\beta$-catenin is transported into the nucleus, where it binds to the LEF/TCF transcription factor and promotes the downstream gene transcription of this pathway [48, 49]. Accumulating evidence suggests that glucocorticoids inactivate Wnt signaling through different mechanisms and further affect the osteogenic and adipogenic differentiation of BMSCs [9, 50-52]. In this study, we identified that PTPN6 acted as the downstream target of METTL14 in BMSCs by performing mRNA sequencing. METTL14 enhanced the stability of PTPN6 mRNA in an m6A-dependent manner, thus promoting the expression of PTPN6. Furthermore, METTL14 promoted the translocation of $\beta$-catenin into the nucleus through the interaction of PTPN6 and GSK-3 $\beta$, and ultimately accelerated the activation of Wnt signal transduction.

In summary, our results demonstrated that the m6A methyltransferase METTL14 can promote proliferation and osteogenic differentiation of SONFH BMSCs by up-regulating the m6A level of PTPN6 and activating the Wnt signaling pathway. These findings suggest that the METTL14-PTPN6 axis may be a potential target for the treatment of SONFH.

\section{MATERIALS AND METHODS}

\section{Patients}

From January 2019 to December 2019, 20 patients with steroid-associated osteonecrosis of the femoral head who underwent hip arthroplasty at the Department of Orthopedics of Henan Provincial People's Hospital were selected as the SONFH group and 20 patients with femoral neck fracture who underwent hip arthroplasty served as the control group. Bone marrow and femoral head tissues were collected during the operation. Table 1 summarizes the clinical characteristics of all patients. The present study was performed in accordance with the ethical standards as laid down in the 1964 Declaration of Helsinki and its later amendments or comparable ethical standards, and this study was approved by the Ethics Committee of Henan Provincial People's Hospital (Approval number:
2017237). All patients signed informed consent before operation.

\section{Bone marrow separation and cell culture}

Five milliliters of bone marrow were procured from the proximal femur during hip arthroplasty. BMSCs were isolated and cultured as described in previous study [12]. Briefly, mononuclear cells in the bone marrow were centrifuged with an equal volume of $1.073 \mathrm{~g} / \mathrm{ml}$ Percoll solution (Sigma, USA) at $2000 \mathrm{rpm}$ for 30 minutes. Then, cells were collected, suspended in DMEM/F12 medium (HyClone, USA) containing 10\% fetal bovine serum (Gibco, USA) and $1 \%$ penicillinstreptomycin (Biosharp, China), and cultured at $37^{\circ} \mathrm{C}$, $5 \% \mathrm{CO}_{2}$ and $99 \%$ relative humidity. In order to maintain the consistent biological characteristics, experiments were carried out when the cells were subcultured to the second generation and were no longer used after they were subcultured to the fifth passage.

\section{Flow cytometry}

In order to verify that cells we extracted were BMSCs, we detected the expression of cell surface markers by flow cytometry. Briefly, we collected cells at the second passage and resuspended them in PBS. Then, $2 \times 10^{5}$ cells were incubated with PE or FITC labeled cell surface marker antibodies against CD29, CD34, CD44 and CD45 (BD Biosciences, USA), as well as isotype control antibody at $4{ }^{\circ} \mathrm{C}$ for 30 minutes in the dark. After the incubation is completed, the samples were analyzed by the flow cytometer (BD Biosciences, USA).

\section{Cell transfection}

The METTL14 overexpression plasmid (termed OEMETTL14) and the negative control (termed OE-NC) were purchased from OBIO Technology (Shanghai, China). Short hairpin RNA targeting PTPN6 (termed sh-PTPN6) and the negative control (termed sh-NC) were designed by GeneChem (Shanghai, China) and cloned into GV112 lentiviral vectors (GeneChem, Shanghai). The cells were infected with the viruses in the presence of Polybrene for 48 hours. Then, positively transfected cells were selected for 7-10 days using 2 $\mu \mathrm{g} / \mathrm{ml}$ puromycin. The overexpression and knockdown efficiencies were determined by qRT-PCR and western blot assay.

\section{Osteogenic and adipogenic differentiation}

For osteogenic differentiation, BMSCs were cultured in complete DMEM/F12 medium containing $0.25 \mathrm{mM}$ ascorbic acid, $10 \mathrm{mM} \beta$-glycerophosphate and $10 \mathrm{nM}$ dexamethasone for 21 days. 
Table 1. Clinical characteristics of patients.

\begin{tabular}{lccc}
\hline & SONFH $(\boldsymbol{n}=\mathbf{2 0})$ & Control $(\boldsymbol{n}=\mathbf{2 0})$ & $\boldsymbol{p}$-value \\
\hline Age (years) & $49.8 \pm 7.6$ & $52.1 \pm 8.4$ & 0.22 \\
Gender (Male/Female) & $11 / 9$ & $10 / 10$ & 0.77 \\
Body Mass Index $\left(\mathrm{kg} / \mathrm{m}^{2}\right)$ & $26.1 \pm 3.2$ & $24.3 \pm 2.7$ & 0.18 \\
Glucocorticoid Medication & $20(100 \%)$ & $1(5 \%)$ & $<0.001$ \\
Total Cholesterol $(\mathrm{mmol} / \mathrm{L})$ & $6.23 \pm 0.85$ & $4.68 \pm 0.72$ & $<0.01$ \\
\hline
\end{tabular}

Adipogenic differentiation of human BMSCs was performed according to an earlier study [53]. Cells were cultured in adipogenesis-inducing medium: complete DMEM/F12 medium containing $1 \mu \mathrm{M}$ dexamethasone, $0.2 \mathrm{mM}$ indomethacin, $0.5 \mathrm{mM}$ isobutyl-methylxanthine and $0.01 \mathrm{mg} / \mathrm{ml}$ insulin. Three days later, the adipogenesis-inducing medium was changed to adipogenesis maintenance medium (complete DMEM/F12 medium containing $0.01 \mathrm{mg} / \mathrm{ml}$ insulin). After 24 hours, switched back to adipogenesis-inducing medium. The cells were alternately cultured in the two media for 3 cycles, after which the cells were maintained in the maintenance medium for one week.

\section{Alizarin red S staining and oil red $\mathrm{O}$ staining}

The cells induced by osteogenic differentiation or adipogenic differentiation were fixed with polyformaldehyde for 30 minutes and then stained with $40 \mathrm{nM}$ alizarin red solution $(\mathrm{pH} 4.2)$ or filtered oil red $\mathrm{O}$ solution at room temperature for 30 minutes. The nonspecific staining was removed with PBS. Stained BMSCs were observed and photographed by an inverted microscope (Olympus, Japan).

\section{Alkaline phosphatase (ALP) activity assay}

ALP activity assay was performed with the Alkaline Phosphatase Assay Kit (Abcam, UK). Initially, 1\% Triton X-100 was added to lyse cells for 20 minutes, followed by centrifugation at $10000 \mathrm{~g}$ for 5 minutes, and the supernatant was collected. Thereafter, the reaction mixture was added and incubated at room temperature for 60 minutes. Finally, the assay buffer was added and incubated again for 30 minutes, the absorbance at $450 \mathrm{~nm}$ was measured by a microplate reader (Thermo Scientific, USA).

\section{Cell proliferation assay}

Cell Counting Kit-8 (CCK-8, Beyotime, China) was used to measure the cell proliferation activity according to the instructions. In brief, the cells were cultured in 96-well plates for 0, 24, 48 and 72 hours and then exchanged for $100 \mu \mathrm{l}$ serum-free medium, and $10 \mu \mathrm{lCCK}-8$ solution was added to each well.
The absorbance at $450 \mathrm{~nm}$ was measured by a microplate reader after incubation at $37^{\circ} \mathrm{C}$ for 2 hours.

\section{EdU assay}

According to the precedent study described [54], the EdU assay kit (RiboBio, China) was adopted to determine the cell proliferation ability. Cells were seeded into 96-well plates at a density of $5 \times 10^{5}$ cells per well, and cultured under standard conditions for 72 hours. Then, they were incubated with EdU buffer at $37^{\circ} \mathrm{C}$ for 2 hours, fixed with $4 \%$ formaldehyde for 30 minutes and permeabilized with $0.1 \%$ Triton X-100 for 20 minutes. EdU solution was added to the culture, followed by the staining of nuclei with DAPI. The results were visualized by a fluorescence microscope (Olympus, Japan).

\section{Quantitative real-time PCR}

Total RNA in femoral head tissues and BMSCs was extracted using TRIzol Reagent and used to synthesize cDNA with the One-Step RT-PCR Kit (Thermo Scientific, USA). qRT-PCR was performed using the SYBR Primer-Script RT-PCR Kit (TaKaRa, Japan) with a CFX96 Touch quantitative PCR system (BioRad, USA). Primers were generated by Sangon Biotechnology (Shanghai, China), and the primer sequences were as follows: METTL14, 5'AGAAACTTGCAGGGCTTCCT-3' (forward) and 5'TCTTCTTCATATGGCAAATTTTCTT-3' (reverse); PTPN6, 5'-GCCTGGACTGTGACATTGAC-3' (forward) and 5'-ATGTTCCCGTACTCCGACTC-3' (reverse); METTL3, 5'-CAAGCTGCACTTCAGAC GAA-3' (forward) and 5'-GCTTGGCGTGTGGT CTTT-3' (reverse); WTAP, 5'-ACGCAGGGAGAAC ATTCTTG-3' (forward) and 5'-CACACTCGGCTGC TGAACT-3' (reverse); FTO, 5'-TTCATGCTGGAT GACCTCAATG-3' (forward) and 5'-GCCAACTGAC AGCGTTCTAAG-3' (reverse); ALKBH5, 5'-TCACT GCATACGGCCTCAGGACAT-3' (forward) and 5'-T TAGAGCAGGGTCCCTGTTGT-3' (reverse); GAPDH, 5' (forward) and 5'-CCAGTAGAGGCAGGGATGAT-3' (reverse). 


\section{Western blot}

Total protein was extracted with RIPA lysate containing cocktail protease inhibitor (Beyotime, China), and nuclear protein was extracted by the CelLytic NuClear Extraction Kit (Sigma, USA). The protein concentration was detected with the BCA Protein Assay Kit (Beyotime, China). Protein lysates were separated by $12 \%$ SDS-PAGE and transferred onto PVDF membranes. Then, PVDF membranes were blocked with 5\% nonfat milk and incubated with primary antibodies. The following primary antibodies were used in this study: anti-METTL14 (ab252562, Abcam), anti-PTPN6 (ab227503, Abcam), anti-RUNX2 (ab76956, Abcam), antiPPAR $\gamma$ (ab59256, Abcam), anti-C/EBP $\alpha$ (ab40764, Abcam), anti-C/EBP $\beta$ (ab15049, Abcam), anti- $\beta$ catenin (ab6302, Abcam), anti-p- $\beta$-catenin (ab11350, Abcam), anti-GSK-3 $\beta$ (ab32391, Abcam), anti-pGSK-3 $\beta$ (ab68476, Abcam), anti-GAPDH (ab8245, Abcam), anti-Histone H3 (ab1791, Abcam). GAPDH and $\mathrm{H} 3$ were used as the internal controls for total protein and nuclear protein, respectively. Horseradish peroxidase-conjugated secondary antibody was used to develop the blots. Blots were detected with the ECL Western Blotting Substrate Kit (ab65623, Abcam) on an ECL system (Bio-Rad, USA).

\section{m6A RNA methylation quantification}

The content of m6A in total RNA was measured by the m6A RNA Methylation Assay Kit (ab185912, Abcam). Briefly, isolated total RNA and bound RNA to the assay wells, and the wells were washed and incubated with capture antibody. After that, rewashed wells and added detection antibody and enhancer solution. Finally, the color developing solution was added and measured the absorbance at $450 \mathrm{~nm}$ by a microplate reader.

\section{mRNA sequencing}

The high-quality total RNA was extracted using TRIzol reagents from $1 \times 10^{6}$ METTL14overexpressing cells and control cells per duplicate and genomic DNA removed using a DNA-free Kit (ThermoFisher, USA). Library construction was conducted using a Collibri 3' mRNA Library Preparation Kit (ThermoFisher, USA) according to the manufacturer's instructions. Quality control and sequencing were performed by Genenergy BioTechnology Inc. The DEGseq software package was used to identify differentially expressed genes between the METTL14 overexpression group and the control group with parameters $p$-value $<0.05$ and false discovery rate $<0.05$.

\section{MeRIP-qPCR}

The m6A level of the individual gene was determined by MeRIP-qPCR assay. According to the previous study [41], purified poly (A) RNA from total RNA was obtained using the Dynabeads mRNA Purification Kit (Thermo Scientific, USA). A/G immunomagnetic beads were incubated with anti-m6A antibody (ab208577, Abcam) or anti-IgG antibody (ab172730, Abcam) in the immunoprecipitation buffer to immunoprecipitate m6ARNA complexes. Thereafter, the complexes were digested by Proteinase $\mathrm{K}(20 \mathrm{mg} / \mathrm{ml})$, and the methylated mRNA of PTPN6 was measured by qRTPCR.

\section{RNA stability}

To evaluate the effect of METTL14 on the stability of PTPN6 mRNA, we detected the half-life of PTPN6 mRNA. Actinomycin D $(5 \mu \mathrm{g} / \mathrm{ml})$ was used to intervene in OE-NC and OE-METTL14 transfected BMSCs from SONFH patients, and total RNA was extracted at 0,2 , 4, 6 and 8 hours. The level of PTPN6 mRNA was measured by qRT-PCR, and the half-life was obtained by nonlinear regression analysis.

\section{Luciferase reporter assay}

Luciferase reporters were generated by cloning wild type 3'UTR of PTPN6 mRNA or mutant type 3'UTR of PTPN6 mRNA (mutant $A$ to $T$ at position 859) into psiCHECK2 (Promega, USA). Then, cells were seeded in 24-well plates and co-transfected with $150 \mathrm{ng}$ of renilla reporters and OE-METTL14. 48 hours later, collected cells and luciferase activities were detected using the Dual-Luciferase Reporter Assay System (Promega, USA) according to manufacturer's protocol.

\section{TOP/FOP-Flash reporter assay}

TOP/FOP-Flash reporter assays were used to determine the transcriptional activity of the $\mathrm{Wnt} / \beta$-catenin pathway. BMSCs were seeded in 24-well plates and transfected with the TOP/FOP-Flash reporter (Biovector NTCC Inc., China). After 48 hours, the luciferase activity was measured with the Dual-Luciferase Reporter Assay System (Promega, USA). The luciferase activity of each sample was normalized to the respective Renilla luciferase activity.

\section{Immunofluorescence staining}

Cells cultured on cover slips were washed twice with cold PBS and fixed with paraformaldehyde for 15 minutes at room temperature and were permeabilized with $0.1 \%$ Triton $\mathrm{X}$ for 20 minutes. Cells were 
incubated with $1 \%$ bovine serum albumin for 40 minutes to block the nonspecific antibody binding sites and subsequently incubated with anti- $\beta$-catenin antibodies $(1: 200)$ overnight at $4^{\circ} \mathrm{C}$. Afterwards, the cells were washed three times with PBS and incubated with a 1:500 dilution of an APC-conjugated secondary antibody for 1 hour. Finally, cells were stained with DAPI to visualize the nuclei. Images were captured by a fluorescence microscope.

\section{IHC staining}

The femoral head tissues were decalcified at room temperature in $15 \%$ EDTA for 5 weeks. After decalcification, the sample was embedded in paraffin. Four micrometer paraffin slides were prepared on glass slides coated with polylysine and then dried for one hour at $60^{\circ} \mathrm{C}$. The slides were blocked with $5 \% \mathrm{BSA}$ and then stained with anti-METTL14 antibody (1:100) or anti-PTPN6 antibody $(1: 100)$ at $4^{\circ} \mathrm{C}$ overnight. Finally, the slides were incubated with HRP-labeled secondary antibody. The slides were visualized by an inverted microscope.

\section{Co-immunoprecipitation assay}

Total protein lysates for immunoprecipitation assay were extracted with IP-lysis buffer (ThermoFisher, USA). The supernatant was collected by centrifugation at $12000 \mathrm{rpm}$ for 10 minutes at $4^{\circ} \mathrm{C}$. For immunoprecipitation, the supernatant was mixed with anti-PTPN6 antibody (ab227503, Abcam) or anti-IgG antibody (ab172730, Abcam) and $50 \mu \mathrm{L}$ of protein $\mathrm{A} / \mathrm{G}$ Dynabeads (ThermoFisher, USA) at $4^{\circ} \mathrm{C}$ overnight. After that, the beads were washed three times with precooled IP lysis buffer, and immunoprecipitates were subjected to western blotting.

\section{Statistical analysis}

All experiments were independently repeated at least three times. Data in this study are expressed as the mean \pm SD. GraphPad Prism 7.0 software was used for all data analysis. The difference between two groups was analyzed by $t$-test. One-way analysis of variance (ANOVA) was used for comparison among the multiple groups. The difference of clinical features between SONFH group and control group was analyzed via $\chi^{2}$ analysis. The linear association between METTL14 and PTPN6 was tested by Pearson's Correlation Coefficient. $P$-value $<0.05$ was considered statistically significant.

\section{ABBREVIATIONS}

BMSC: bone marrow mesenchymal stem cell; SONFH: steroid-associated osteonecrosis of the femoral head;
m6A: N6-methyladenosine; METTL14: methyltransferase 14; PTPN6: protein tyrosine phosphatase non-receptor type 6; GSK-3 $\beta$ : glycogen

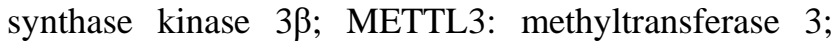
WTAP: WT1 associated protein; FTO: FTO alphaketoglutarate dependent dioxygenase; ALKBH5: alkB homolog 5; DMEM: Dulbecco's modified Eagle's medium; ALP: alkaline phosphatase; EdU: 5-ethynyl2'-deoxyuridine; CCK-8: cell counting kit-8; qRT-PCR: quantitative real-time polymerase chain reaction; Co-IP: co-immunoprecipitation; MeRIP: RNA methylation immunoprecipitation; IHC: immunohistochemistry; ANOVA: one-way analysis of variance.

\section{AUTHOR CONTRIBUTIONS}

Cheng Cheng and Haoping Zhang were involved in the execution of experiments, data analysis, and manuscript writing; Jia Zheng, Yi Jin and Donghui Wang were all involved in the collection of clinical specimens; Zhipeng Dai was involved in the design and supervision of experiments, and revised the manuscript. All authors provided final approval of the version to be submitted.

\section{CONFLICTS OF INTEREST}

The authors declare no conflicts of interest related to this study.

\section{FUNDING}

This work was supported by grants from the National Natural Science Foundation of China (grant number: 82002300).

\section{REFERENCES}

1. Karol SE, Yang W, Van Driest SL, Chang TY, Kaste S, Bowton E, Basford M, Bastarache L, Roden DM, Denny JC, Larsen E, Winick N, Carroll WL, et al. Genetics of glucocorticoid-associated osteonecrosis in children with acute lymphoblastic leukemia. Blood. 2015; 126:1770-6. https://doi.org/10.1182/blood-2015-05-643601 PMID:26265699

2. Wang $\mathrm{C}$, Meng $\mathrm{H}$, Wang $\mathrm{X}$, Zhao C, Peng J, Wang $\mathrm{Y}$. Differentiation of Bone Marrow Mesenchymal Stem Cells in Osteoblasts and Adipocytes and its Role in Treatment of Osteoporosis. Med Sci Monit. 2016; 22:226-33.

https://doi.org/10.12659/msm.897044 PMID:26795027

3. Chen $Q$, Shou $P$, Zheng $C$, Jiang $M$, Cao $G$, Yang $Q$, Cao J, Xie N, Velletri T, Zhang X, Xu C, Zhang L, Yang H, et al. Fate decision of mesenchymal stem cells: 
adipocytes or osteoblasts? Cell Death Differ. 2016; 23:1128-39.

https://doi.org/10.1038/cdd.2015.168

PMID:26868907

4. Motegi SI, Ishikawa O. Mesenchymal stem cells: The roles and functions in cutaneous wound healing and tumor growth. J Dermatol Sci. 2017; 86:83-9. https://doi.org/10.1016/i.jdermsci.2016.11.005 PMID:27866791

5. Chang YH, Liu HW, Wu KC, Ding DC. Mesenchymal Stem Cells and Their Clinical Applications in Osteoarthritis. Cell Transplant. 2016; 25:937-50. https://doi.org/10.3727/096368915X690288 PMID:26688464

6. Crane JL, Cao X. Bone marrow mesenchymal stem cells and TGF- $\beta$ signaling in bone remodeling. J Clin Invest. 2014; 124:466-72. https://doi.org/10.1172/JCl70050 PMID:24487640

7. Wang $X$, Wang $Y$, Gou W, Lu Q, Peng J, Lu S. Role of mesenchymal stem cells in bone regeneration and fracture repair: a review. Int Orthop. 2013; 37:2491-8.

https://doi.org/10.1007/s00264-013-2059-2 PMID:23948983

8. Li X, Wang M, Jing X, Guo W, Hao C, Zhang Y, Gao S, Chen $M$, Zhang Z, Zhang X, Shen S, Zhang B, Xian H, et al. Bone Marrow- and Adipose Tissue-Derived Mesenchymal Stem Cells: Characterization, Differentiation, and Applications in Cartilage Tissue Engineering. Crit Rev Eukaryot Gene Expr. 2018; 28:285-310.

https://doi.org/10.1615/CritRevEukaryotGeneExpr.20 18023572 PMID:30311578

9. Han L, Wang B, Wang R, Gong S, Chen G, Xu W. The shift in the balance between osteoblastogenesis and adipogenesis of mesenchymal stem cells mediated by glucocorticoid receptor. Stem Cell Res Ther. 2019; 10:377.

https://doi.org/10.1186/s13287-019-1498-0 PMID:31805987

10. Dai Z, Jin Y, Zheng J, Liu K, Zhao J, Zhang S, Wu F, Sun Z. MiR-217 promotes cell proliferation and osteogenic differentiation of BMSCs by targeting DKK1 in steroidassociated osteonecrosis. Biomed Pharmacother. 2019; 109:1112-9. https://doi.org/10.1016/i.biopha.2018.10.166 PMID:30551361

11. Zhang F, Peng W, Zhang J, Dong $W$, Wu J, Wang $T$, Xie Z. P53 and Parkin co-regulate mitophagy in bone marrow mesenchymal stem cells to promote the repair of early steroid-induced osteonecrosis of the femoral head. Cell Death Dis. 2020; 11:42.

https://doi.org/10.1038/s41419-020-2238-1 PMID:31959744

12. Hao C, Yang S, Xu W, Shen JK, Ye S, Liu X, Dong Z, Xiao $B$, Feng Y. MiR-708 promotes steroid-induced osteonecrosis of femoral head, suppresses osteogenic differentiation by targeting SMAD3. Sci Rep. 2016; 6:22599.

https://doi.org/10.1038/srep22599

PMID:26932538

13. Wu X, Yang S, Wang H, Meng C, Xu W, Duan D, Liu X. G-CSF/SCF exert beneficial effects via anti-apoptosis in rabbits with steroid-associated osteonecrosis. Exp Mol Pathol. 2013; 94:247-54.

https://doi.org/10.1016/j.yexmp.2012.06.003 PMID:22750282

14. Sun ZB, Wang JW, Xiao H, Zhang QS, Kan WS, Mo $\mathrm{FB}$, $\mathrm{Hu} \mathrm{S}$, Ye SN. Icariin may benefit the mesenchymal stem cells of patients with steroidassociated osteonecrosis by ABCB1-promoter demethylation: a preliminary study. Osteoporos Int. 2015; 26:187-97. https://doi.org/10.1007/s00198-014-2809-z PMID:25112719

15. Zhao BS, Roundtree IA, He C. Post-transcriptional gene regulation by mRNA modifications. Nat Rev Mol Cell Biol. 2017; 18:31-42.

https://doi.org/10.1038/nrm.2016.132 PMID:27808276

16. Yue Y, Liu J, He C. RNA N6-methyladenosine methylation in post-transcriptional gene expression regulation. Genes Dev. 2015; 29:1343-55. https://doi.org/10.1101/gad.262766.115 PMID:26159994

17. Meyer KD, Jaffrey SR. Rethinking $m^{6} A$ Readers, Writers, and Erasers. Annu Rev Cell Dev Biol. 2017; 33:319-42.

https://doi.org/10.1146/annurev-cellbio-100616060758

PMID:28759256

18. Lence T, Paolantoni C, Worpenberg L, Roignant JY. Mechanistic insights into $\mathrm{m}^{6}$ A RNA enzymes. Biochim Biophys Acta Gene Regul Mech. 2019; 1862:222-9. https://doi.org/10.1016/i.bbagrm.2018.10.014 PMID:30395944

19. Chen $X Y$, Zhang J, Zhu JS. The role of $m^{6}$ A RNA methylation in human cancer. Mol Cancer. 2019; 18:103.

https://doi.org/10.1186/s12943-019-1033-z PMID:31142332 
20. Roundtree IA, Evans ME, Pan T, He C. Dynamic RNA Modifications in Gene Expression Regulation. Cell. 2017; 169:1187-200.

https://doi.org/10.1016/i.cell.2017.05.045

PMID:28622506

21. Fu Y, Dominissini D, Rechavi G, He C. Gene expression regulation mediated through reversible $\mathrm{m}^{6}$ A RNA methylation. Nat Rev Genet. 2014; 15:293-306.

https://doi.org/10.1038/nrg3724

PMID:24662220

22. Wang $X$, Zhao BS, Roundtree IA, Lu Z, Han D, Ma H, Weng X, Chen K, Shi H, He C. N(6)-methyladenosine Modulates Messenger RNA Translation Efficiency. Cell. 2015; 161:1388-99.

https://doi.org/10.1016/i.cell.2015.05.014 PMID:26046440

23. Feng $Z$, Li $Q$, Meng $R, Y i B, X u Q$. METTL3 regulates alternative splicing of MyD88 upon the lipopolysaccharide-induced inflammatory response in human dental pulp cells. J Cell Mol Med. 2018; 22:2558-68.

https://doi.org/10.1111/jcmm.13491

PMID:29502358

24. Wang X, Lu Z, Gomez A, Hon GC, Yue Y, Han D, Fu Y, Parisien M, Dai $Q$, Jia $G$, Ren B, Pan T, He C. N6methyladenosine-dependent regulation of messenger RNA stability. Nature. 2014; 505:117-20.

https://doi.org/10.1038/nature 12730 PMID:24284625

25. Widagdo J, Anggono V. The m6A-epitranscriptomic signature in neurobiology: from neurodevelopment to brain plasticity. J Neurochem. 2018; 147:137-52.

https://doi.org/10.1111/inc.14481

PMID:29873074

26. Wang $S$, Chai $P$, Jia $R$, Jia R. Novel insights on $m^{6} A$ RNA methylation in tumorigenesis: a double-edged sword. Mol Cancer. 2018; 17:101.

https://doi.org/10.1186/s12943-018-0847-4

PMID:30031372

27. Wei W, Ji X, Guo X, Ji S. Regulatory Role of $\mathrm{N}^{6}$ methyladenosine $\left(m^{6}\right.$ A) Methylation in RNA Processing and Human Diseases. J Cell Biochem. 2017; 118:2534-43.

https://doi.org/10.1002/jcb.25967

PMID:28256005

28. Wu $Y$, Xie L, Wang $M$, Xiong $Q$, Guo $Y$, Liang $Y$, Li J, Sheng R, Deng P, Wang $Y$, Zheng R, Jiang $Y$, Ye L, et al. Mettl3-mediated $\mathrm{m}^{6} \mathrm{~A}$ RNA methylation regulates the fate of bone marrow mesenchymal stem cells and osteoporosis. Nat Commun. 2018; 9:4772. https://doi.org/10.1038/s41467-018-06898-4 PMID:30429466
29. Liu J, Chen M, Ma L, Dang X, Du G. piRNA-36741 regulates $B M P 2-$ mediated osteoblast differentiation via METTL3 controlled m6A modification. Aging (Albany NY). 2021; 13:23361-75.

https://doi.org/10.18632/aging.203630

PMID:34645714

30. Xie Z, Yu W, Zheng G, Li J, Cen S, Ye G, Li Z, Liu W, Li $M$, Lin J, Su Z, Che Y, Ye F, et al. TNF- $\alpha$-mediated $m^{6} A$ modification of ELMO1 triggers directional migration of mesenchymal stem cell in ankylosing spondylitis. Nat Commun. 2021; 12:5373.

https://doi.org/10.1038/s41467-021-25710-4 PMID:34508078

31. Wang J, Fu Q, Yang J, Liu JL, Hou SM, Huang X, Cao JS, Liu TL, Wang KZ. RNA N6-methyladenosine demethylase FTO promotes osteoporosis through demethylating Runx2 mRNA and inhibiting osteogenic differentiation. Aging (Albany NY). 2021; 13:21134-41.

https://doi.org/10.18632/aging.203377

PMID: $\underline{34496349}$

32. Forbes K, Skinner L, Aplin JD, Westwood M. The tyrosine phosphatase SHP-1 negatively regulates cytotrophoblast proliferation in first-trimester human placenta by modulating EGFR activation. Cell Mol Life Sci. 2012; 69:4029-40.

https://doi.org/10.1007/s00018-012-1067-5 PMID:22797910

33. Li X, Yang H, Wu S, Meng Q, Sun H, Lu R, Cui J, Zheng $Y$, Chen $W$, Zhang $R$, Aschner $M$, Chen $R$. Suppression of PTPN6 exacerbates aluminum oxide nanoparticle-induced COPD-like lesions in mice through activation of STAT pathway. Part Fibre Toxicol. 2017; 14:53.

https://doi.org/10.1186/s12989-017-0234-0

PMID:29233151

34. Xu E, Forest MP, Schwab M, Avramoglu RK, St-Amand E, Caron AZ, Bellmann K, Shum M, Voisin G, Paquet $M$, Montoudis $A$, Lévy $E$, Siminovitch $K A$, et al. Hepatocyte-specific Ptpn6 deletion promotes hepatic lipid accretion, but reduces NAFLD in diet-induced obesity: potential role of PPARy. Hepatology. 2014; 59:1803-15.

https://doi.org/10.1002/hep.26957 PMID:24327268

35. Maeda K, Kobayashi Y, Koide M, Uehara S, Okamoto M, Ishihara A, Kayama T, Saito M, Marumo K. The Regulation of Bone Metabolism and Disorders by Wnt Signaling. Int J Mol Sci. 2019; 20:5525.

https://doi.org/10.3390/ijms20225525 PMID: $\underline{31698687}$

36. Lerner UH, Ohlsson C. The WNT system: background and its role in bone. J Intern Med. 2015; 277:630-49. 
https://doi.org/10.1111/joim.12368

PMID:25845559

37. Tan Z, Ding N, Lu H, Kessler JA, Kan L. Wnt signaling in physiological and pathological bone formation. Histol Histopathol. 2019; 34:303-12.

https://doi.org/10.14670/HH-18-062 PMID: 30422303

38. Li R, Lin $Q X$, Liang $X Z$, Liu GB, Tang $H$, Wang $Y$, Lu SB, Peng J. Stem cell therapy for treating osteonecrosis of the femoral head: From clinical applications to related basic research. Stem Cell Res Ther. 2018; 9:291.

https://doi.org/10.1186/s13287-018-1018-7 PMID:30359305

39. Zhang C, Fu J, Zhou Y. A Review in Research Progress Concerning m6A Methylation and Immunoregulation. Front Immunol. 2019; 10:922.

https://doi.org/10.3389/fimmu.2019.00922 PMID:31080453

40. Weng $H$, Huang $H$, Wu $H$, Qin $X$, Zhao BS, Dong L, Shi $\mathrm{H}$, Skibbe J, Shen $\mathrm{C}$, Hu C, Sheng $\mathrm{Y}$, Wang $\mathrm{Y}$, Wunderlich $\mathrm{M}$, et al. METTL14 Inhibits Hematopoietic Stem/Progenitor Differentiation and Promotes Leukemogenesis via mRNA $\mathrm{m}^{6} \mathrm{~A}$ Modification. Cell Stem Cell. 2018; 22:191-205.e9.

https://doi.org/10.1016/i.stem.2017.11.016 PMID:29290617

41. Ma JZ, Yang F, Zhou CC, Liu F, Yuan JH, Wang F, Wang TT, Xu QG, Zhou WP, Sun SH. METTL14 suppresses the metastatic potential of hepatocellular carcinoma by modulating $\mathrm{N}^{6}$-methyladenosine-dependent primary MicroRNA processing. Hepatology. 2017; 65:529-43.

https://doi.org/10.1002/hep.28885

PMID:27774652

42. López-Ruiz $P$, Rodriguez-Ubreva J, Cariaga $A E$, Cortes MA, Colás B. SHP-1 in cell-cycle regulation. Anticancer Agents Med Chem. 2011; 11:89-98. https://doi.org/10.2174/187152011794941154 PMID:21291405

43. Kanwal Z, Zakrzewska A, den Hertog J, Spaink HP, Schaaf MJ, Meijer AH. Deficiency in hematopoietic phosphatase ptpn6/Shp1 hyperactivates the innate immune system and impairs control of bacterial infections in zebrafish embryos. J Immunol. 2013; 190:1631-45.

https://doi.org/10.4049/jimmunol.1200551

PMID:23335748

44. Zhang L, Oh SY, Wu X, Oh MH, Wu F, Schroeder JT, Takemoto CM, Zheng T, Zhu Z. SHP-1 deficient mast cells are hyperresponsive to stimulation and critical in initiating allergic inflammation in the lung. J Immunol. 2010; 184:1180-90. https://doi.org/10.4049/jimmunol.0901972 PMID:20042576

45. Jiang M, Zheng C, Shou P, Li N, Cao G, Chen Q, Xu C, Du L, Yang Q, Cao J, Han Y, Li F, Cao W, et al. SHP1 Regulates Bone Mass by Directing Mesenchymal Stem Cell Differentiation. Cell Rep. 2016; 17:2161. https://doi.org/10.1016/i.celrep.2016.10.069 PMID:27851976

46. Steinhart Z, Angers S. Wnt signaling in development and tissue homeostasis. Development. 2018; 145:dev146589.

https://doi.org/10.1242/dev.146589

PMID:29884654

47. Wang Y, Li YP, Paulson C, Shao JZ, Zhang X, Wu M, Chen W. Wnt and the Wnt signaling pathway in bone development and disease. Front Biosci (Landmark Ed). 2014; 19:379-407.

https://doi.org/10.2741/4214

PMID:24389191

48. MacDonald BT, Tamai K, He X. Wnt/beta-catenin signaling: components, mechanisms, and diseases. Dev Cell. 2009; 17:9-26.

https://doi.org/10.1016/i.devcel.2009.06.016 PMID:19619488

49. Clevers $H$, Nusse R. Wnt/ $\beta$-catenin signaling and disease. Cell. 2012; 149:1192-205. https://doi.org/10.1016/j.cell.2012.05.012 PMID:22682243

50. Yuan Z, Li Q, Luo S, Liu Z, Luo D, Zhang B, Zhang D, Rao P, Xiao J. PPARy and Wnt Signaling in Adipogenic and Osteogenic Differentiation of Mesenchymal Stem Cells. Curr Stem Cell Res Ther. 2016; 11:216-25. https://doi.org/10.2174/1574888x10666150519093429 PMID:25986621

51. Guañabens N, Gifre L, Peris P. The role of Wnt signaling and sclerostin in the pathogenesis of glucocorticoid-induced osteoporosis. Curr Osteoporos Rep. 2014; 12:90-7.

https://doi.org/10.1007/s11914-014-0197-0 PMID:24488619

52. Hu X, Gao JH, Liao YJ, Tang SJ, Lu F. Dexamethasone alters epithelium proliferation and survival and suppresses $W n t / \beta$-catenin signaling in developing cleft palate. Food Chem Toxicol. 2013; 56:67-74. https://doi.org/10.1016/i.fct.2013.02.003 PMID:23416130

53. Yu WH, Li FG, Chen XY, Li JT, Wu YH, Huang LH, Wang Z, Li P, Wang T, Lahn BT, Xiang AP. PPARy suppression inhibits adipogenesis but does not promote osteogenesis of human mesenchymal stem cells. Int J Biochem Cell Biol. 2012; 44:377-84. https://doi.org/10.1016/j.biocel.2011.11.013 
PMID:22120652

54. Chen Y, Peng C, Chen J, Chen D, Yang B, He B, Hu W, Zhang Y, Liu H, Dai L, Xie H, Zhou L, Wu J, Zheng S.

WTAP facilitates progression of hepatocellular carcinoma via m6A-HuR-dependent epigenetic silencing of ETS1. Mol Cancer. 2019; 18:127.

https://doi.org/10.1186/s12943-019-1053-8

PMID:31438961 